Article

\title{
Improved DRUS 4H-SiC MESFET with High Power Added Efficiency
}

\author{
Hujun Jia *, Yuan Liang, Tao Li, Yibo Tong, Shunwei Zhu®, Xingyu Wang, Tonghui Zeng and \\ Yintang Yang
}

School of Microelectronics, Xidian University, Xi'an 710071, China; ly_3421@163.com (Y.L.); lit77777@163.com (T.L.); yibo_tong@126.com (Y.T.); swzhu@stu.xidian.edu.cn (S.Z.); 18844504798@163.com (X.W.); zeng_tonghui@163.com (T.Z.); ytyang@xidian.edu.cn (Y.Y.)

* Correspondence: hjjia@mail.xidian.edu.cn; Tel.: +86-137-7212-6387

Received: 22 November 2019; Accepted: 25 December 2019; Published: 27 December 2019

\begin{abstract}
A 4H-SiC metal semiconductor field effect transistor (MESFET) with layered doping and undoped space regions (LDUS-MESFET) is proposed and simulated by ADS and ISE-TCAD software in this paper. The structure (LDUS-MESFET) introduced layered doping under the lower gate of the channel, while optimizing the thickness of the undoped region. Compared with the double-recessed $4 \mathrm{H}-\mathrm{SiC}$ MESFET with partly undoped space region (DRUS-MESFET), the power added efficiency of the LDUS-MESFET is increased by $85.8 \%$, and the saturation current is increased by $27.4 \%$. Although the breakdown voltage of the device has decreased, the decrease is within an acceptable range. Meanwhile, the LDUS-MESFET has a smaller gate-source capacitance and a large transconductance. Therefore, the LDUS-MESFET can better balance DC and AC characteristics and improve power added efficiency (PAE).
\end{abstract}

Keywords: 4H-SiC MESFET; simulation; power added efficiency (PAE)

\section{Introduction}

As a third-generation semiconductor, silicon carbide $(\mathrm{SiC})$ has significant advantages in materials and devices, with high critical electrical field, high thermal conductivity, and high electron saturation velocity [1-3]. SiC based metal-semiconductor field-effect transistors (MESFETs) have become the key components for high-power, high-efficiency and high-frequency microwave applications because of their excellent properties. They offer wider bandwidth operation and lower system size than $\mathrm{Si}$ and GaAs based on MESFET technologies [4-7]. Based on those excellent performance, 4H-SiC MESFETs have broad application prospects in aerospace, satellite communications, and active phased array radars. Previously, the main research on SiC-MESFET was to balance the DC and AC characteristics and improve the output power density. With the idea of energy saving and emission reduction, high efficiency and low energy consumption have become the direction of device design. Thus, some improved structures have been reported for improving the power added efficiency. Such as a novel $4 \mathrm{H}-\mathrm{SiC}$ MESFET with multi-recessed p-buffer layer for high energy-efficiency applications [8], an improved UU-MESFET with high power added efficiency [9], multi-recessed 4H-SiC metal semiconductor field effect transistor (MRD-MESFET) with high power added efficiency [10], an improved 4H-SiC MESFET with a partially low doped channel [11]. However, the trade-off between PAE and DC AC parameters is still a troublesome problem.

The DRUS-MESFET [12] is proposed to increase the breakdown voltage and improve the output power density of the device based on the double-recessed MESFET (DR-MESFET) [13]. But the saturation current and PAE of the device is low. Considering the saturation current and the power added efficiency, an improved double-recessed $4 \mathrm{H}-\mathrm{SiC}$ MESFET with partly undoped space region 
(LDUS-MESFET) is proposed and simulated. The new structure introduced layered doping under the lower gate and optimized the thickness of the undoped region. Using software ISE-TCAD and ADS to simulate the LDUS-MESFET, the DC and AC parameter values and power added efficiency of the device are obtained. By comparing with the DRUS-MESFET, it can be seen that the LDUS-MESFET is more in line with "green high efficiency, energy saving and emission reduction" thoughts.

\section{Device Structure and Simulation Method and Fabrication Feasibility}

\subsection{Device Structure}

Schematic cross-section of the DRUS-MESFET and the LDUS-MESFET are shown in Figure 1a,b, respectively. They both have a semi-insulating substrate, a p-type buffer layer, an n-type channel layer, and two highly doped n-type cap layers. The semi-insulating substrate is modeled as a compensation-doped (vanadium) semiconductor with a high concentration of deep level impurities [13]. Among them, the role of the $\mathrm{p}$ buffer layer is to reduce the influence of substrate defects on the active layer and improve noise performance and device gain [14]. Both of these structures have upper and lower gates, which control a thinner and a thicker part of the channel, respectively. The difference between the two structures is that the LDUS-MESFET has a layered doping under the lower gate, wherein the upper layer region has a doping thickness of $H_{1}=0.05 \mu \mathrm{m}$ and a concentration of $6 \times 10^{17} \mathrm{~cm}^{-3}$ (to have a meaningful comparison, the lower layer region doping concentration of the LDUS-MESFET based on the DRUS-MESFET is also set at $3 \times 10^{17} \mathrm{~cm}^{-3}$ ), and the thickness of the undoped space region (US) is optimized for high efficiency. The optimization result is $\mathrm{H}_{2}=0.05 \mu \mathrm{m}$. The other parameters of the two structures are shown in Table 1.

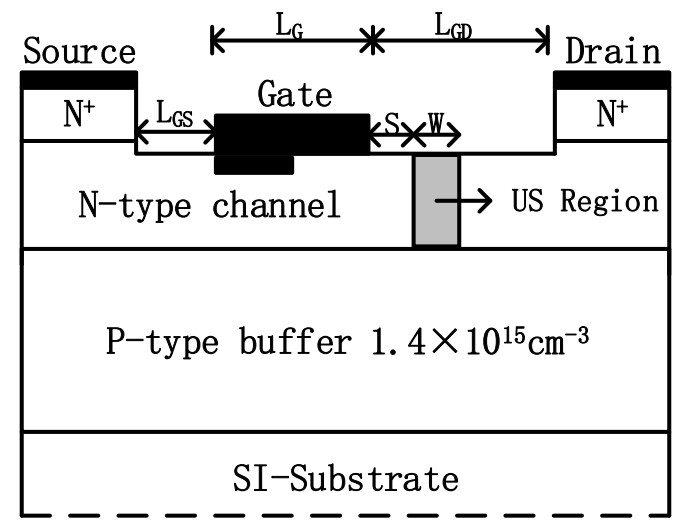

(a)

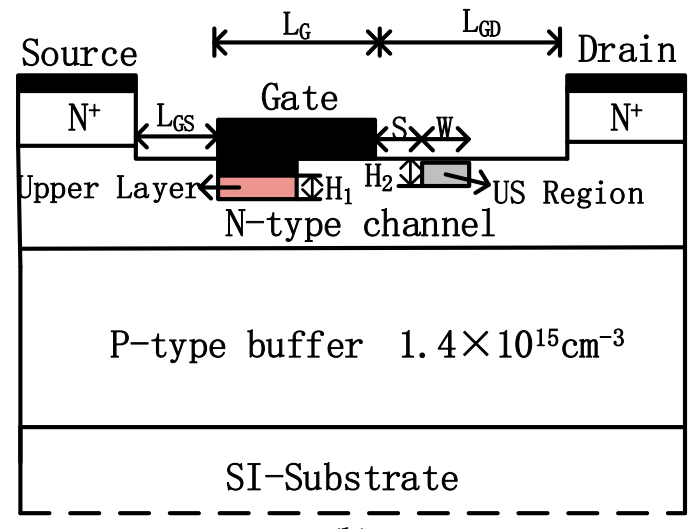

(b)

Figure 1. Schematic cross sections of the (a) double-recessed 4H-SiC MESFET with partly undoped space region (DRUS-MESFET), (b) 4H-SiC metal semiconductor field effect transistor with layered doping and undoped space regions (LDUS-MESFET). 
Table 1. Common parameters of the two structures

\begin{tabular}{cc}
\hline Parameters & Values \\
\hline P-Buffer Concentration & $1.4 \times 10^{15} \mathrm{~cm}^{-3}$ \\
N-Channel Concentration & $3 \times 10^{17} \mathrm{~cm}^{-3}$ \\
N-Cap layers Concentration & $2 \times 10^{19} \mathrm{~cm}^{-3}$ \\
Upper layer Concentration & $6 \times 10^{17} \mathrm{~cm}^{-3}$ \\
N-Cap layers Thickness & $0.2 \mu \mathrm{m}$ \\
N-Channel Thickness & $0.25 \mu \mathrm{m}$ \\
P-Buffer Thickness & $0.5 \mu \mathrm{m}$ \\
Recess gate Thickness & $0.05 \mu \mathrm{m}$ \\
Recess gate Width & $0.35 \mu \mathrm{m}$ \\
$W$ & $0.3 \mu \mathrm{m}$ \\
$S$ & $0.2 \mu \mathrm{m}$ \\
$L_{\mathrm{gs}}$ & $0.5 \mu \mathrm{m}$ \\
$L_{\mathrm{gd}}$ & $1.0 \mu \mathrm{m}$ \\
$L_{\mathrm{s}}$ & $0.5 \mu \mathrm{m}$ \\
$L_{\mathrm{d}}$ & $0.5 \mu \mathrm{m}$ \\
$L_{\mathrm{g}}$ & $0.7 \mu \mathrm{m}$ \\
$H_{1}$ & $0.05 \mu \mathrm{m}$ \\
$H_{2}$ & $0.05 \mu \mathrm{m}$ \\
\hline
\end{tabular}

\subsection{Simulation Method}

Two dimensional numerical device characteristics are realized with ISE-TCAD. The simulator is calibrated with experimental data in the micrometer regime [15], and the agreement between experimental data and simulation results is obtained as shown in Figure 2. In order to accurately simulate the electrical characteristics of the $4 \mathrm{H}-\mathrm{SiC}$ MESFET device structure, the main physical models used are mobility (Enormal, Doping Dep, High Field saturation (GradQuasiFermi)), recombination (Auger, SRH (DopingDep)), incomplete ionization, effective intrinsic density (Band Gap Narrowing (OldSlotboom)). The DC and AC parameters of the device are obtained by ISE-TCAD simulation, and these parameters are input into the ADS software to simulate the power added efficiency of the device. In the ADS simulation, the model used is EE-FET3, an empirical analytical nonlinear model used to fit the electrical properties of MESFETs. And the working bias conditions were set as follows: $V_{\mathrm{gs}}$ was $-3.5 \mathrm{~V}, V_{\mathrm{ds}}$ was $28 \mathrm{~V}$, RF was $1.2 \mathrm{GHz}$ and input power Pavs was $24 \mathrm{dBm}$.

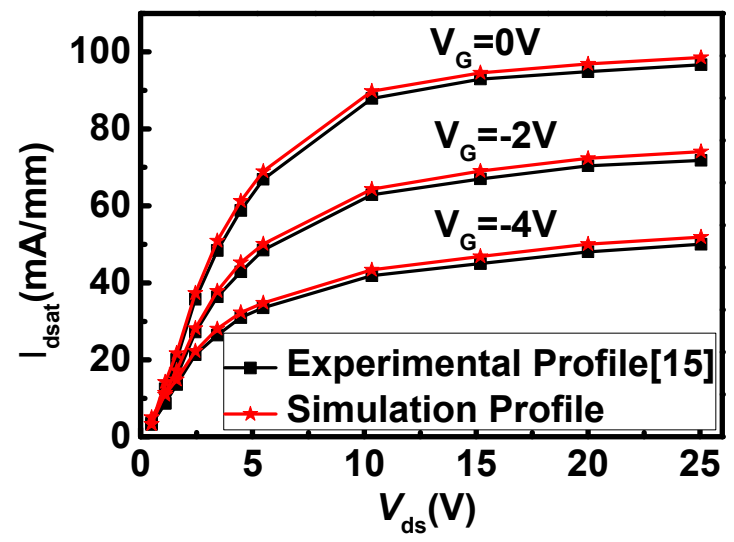

Figure 2. Comparison of experimental data and simulation data on output current.

\subsection{Fabrication Feasibility}

The LDUS-MESFET can be fabricated using the same procedures as reported in [16]. It is worth noting that the doping concentration of $6 \times 10^{17} \mathrm{~cm}^{-3}$ region under the lower gate can be formed by ion implantation and activation process. Through high temperature and multi-energy ion implantation with phosphorous, and activation of the implanted ions can be achieved by inductively heating at a 
desired time and temperature in an Ar atmosphere [17]. The formation of undoped space region is formed by high energy ion implantation of deep level vanadium and high temperature annealing (the resistivity of the US region is $2 \times 10^{6}-7.6 \times 10^{6} \Omega \cdot \mathrm{cm}$ ).

The recessed gate area of the transistors can be fabricated as reported in [16] as follows: First, a thermal oxide layer is deposited on top of the channel, the thermal oxide layer is etched through the position of the recessed area and continues to be etched into the interior of the channel to form a recessed area having a thickness of $0.05 \mu \mathrm{m}$. Second, Nickel with a work function of $5.1 \mathrm{eV}$ was deposited on the recessed area to form a Schottky contact.

\section{Results and Discussion}

\subsection{The Effect of the Doping Concentration $\left(\mathrm{N}_{d}\right)$ and Thickness $\left(\mathrm{H}_{1}\right)$ of the Upper Layer Region on the Device Parameters}

The effect of the thickness and doping concentration of the upper layer region on DC and AC parameters are shown in Figure 3. It can be seen from the Figure $3 a$ that as the doping concentration increases, the absolute value of the threshold voltage increases significantly. When $H_{1}$ is less than $0.05 \mu \mathrm{m}$, the absolute value of Threshold voltage $\left(V_{\mathrm{t}}\right)$ is gradually decreasing with the increase of $H_{1}$. Because there is a longitudinal concentration gradient in the channel. The concentration gradient produces a longitudinal electric field that weakens the pinch-off voltage, which results in a decrease in the absolute value of the threshold voltage. When $H_{1}$ is greater than $0.05 \mu \mathrm{m}$, the absolute value of $V_{\mathrm{t}}$ is increasing with the increase of $H_{1}$. Because as the doping thickness increases, the number of electrons in the channel increases, causing the threshold voltage to drift negatively. At $H_{1}=0.05 \mu \mathrm{m}$, the absolute value of the threshold voltage has a minimum value. In Figure $3 \mathrm{~b}$. As the doping concentration increases, Gate-source capacitance $\left(C_{\mathrm{gs}}\right)$ also increases. At the same time, $C_{\mathrm{gs}}$ shows an upward trend with the increase of $H_{1}$. In Figure $3 c$,d, as the doping concentration increases, the saturation current $\left(I_{\text {dsat }}\right)$ increases significantly, and the breakdown voltage decreases. When $H_{1}$ rises from 0 to $0.05 \mu \mathrm{m}$, the saturation current increases rapidly. When $H_{1}$ increases from $0.05 \mu \mathrm{m}$, the rising trend of saturation current becomes slow.

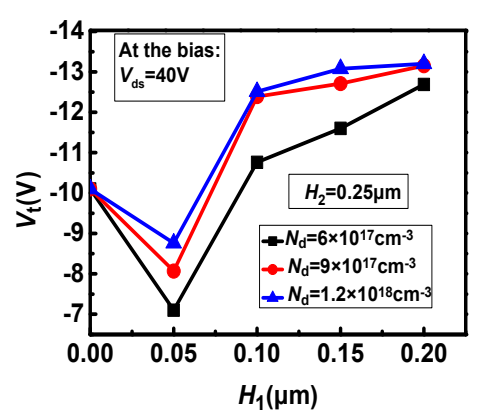

(a)

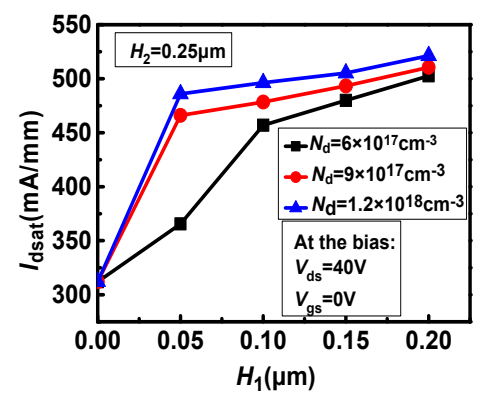

(c)

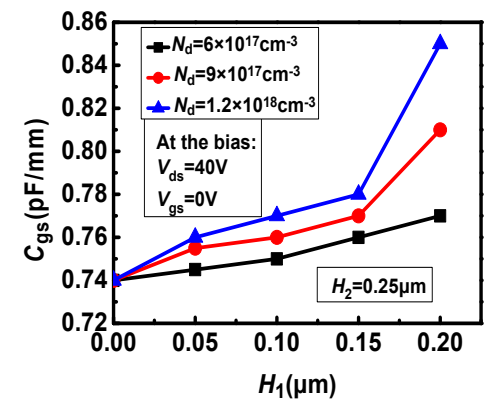

(b)

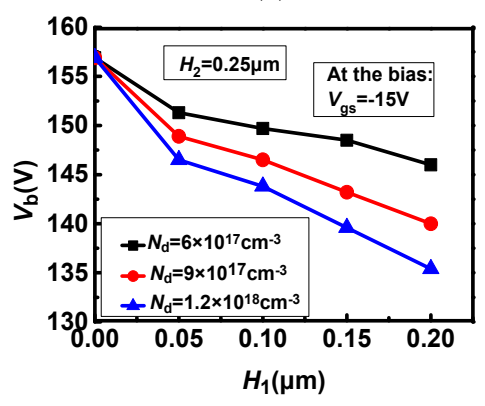

(d)

Figure 3. The effect of $H_{1}$ and $N_{\mathrm{d}}$ on the device parameters: (a) $V_{\mathrm{t}}-N_{\mathrm{d}}$ and $H_{1}$, (b) $C_{\mathrm{gs}}-N_{\mathrm{d}}$ and $H_{1}$, (c) $I_{\text {dsat }}-N_{\mathrm{d}}$ and $H_{1}$, (d) $V_{\mathrm{b}}-N_{\mathrm{d}}$ and $H_{1}$. 


\subsection{The Effect of the Doping Concentration $\left(N_{d}\right)$ and Thickness of the Upper Layer Region $\left(H_{1}\right)$ on PAE}

The influences of the doping concentration and thickness of upper layer region on PAE is shown in Figure 4. It can be seen from the figure that as the doping concentration of the upper layer region increases, the PAE significantly decreases. When the thickness of the upper layer region is less than $0.05 \mu \mathrm{m}$, the PAE increases with the increase of $H_{1}$. When $H_{1}$ is greater than $0.05 \mu \mathrm{m}$, the PAE decreases with the increase of $H_{1}$. When the thickness of the upper layer region is $0.05 \mu \mathrm{m}$, the PAE reaches a maximum value.

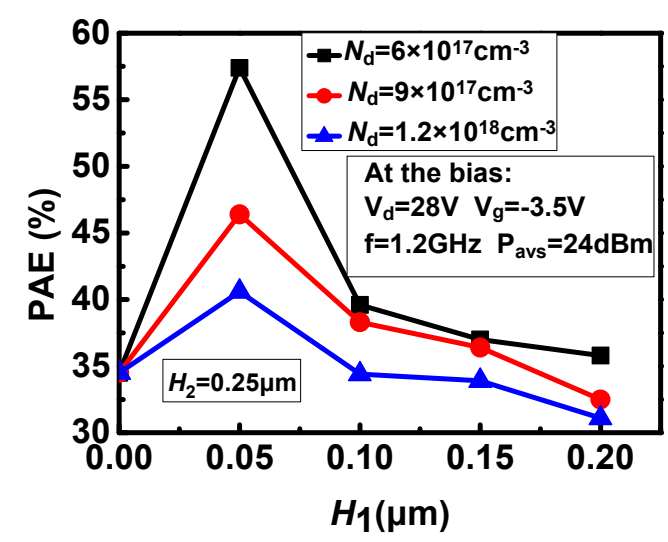

Figure 4. The effects of $N_{\mathrm{d}}$ and $H_{1}$ on the PAE.

\subsection{Optimization of the Undoped Region Thickness $\left(\mathrm{H}_{2}\right)$}

Based on the thickness and doping concentration of the upper layer region determined above, the thickness of the undoped region is optimized to obtain better power added efficiency and DC AC parameters. The effect of the thickness of the undoped region on the DC, AC parameters and PAE of the device is shown in Figure 5. In Figure 5a. We can see that as $\mathrm{H}_{2}$ increases, the absolute value of the threshold voltage gradually decreases. At the same time, when $H_{2}$ is less than $0.05 \mu \mathrm{m}$, the breakdown voltage increases as $\mathrm{H}_{2}$ increases, when $\mathrm{H}_{2}$ is greater than $0.05 \mu \mathrm{m}$, the breakdown voltage shows a basically constant trend with the increase of $\mathrm{H}_{2}$. In Figure $5 \mathrm{~b}$. As $\mathrm{H}_{2}$ increases, the transconductance $\left(g_{\mathrm{m}}\right)$ shows a decreasing trend. When $H_{2}$ is less than $0.15 \mu \mathrm{m}$, the transconductance decline trend is more gradual. When $H_{2}$ is greater than $0.15 \mu \mathrm{m}$, the transconductance decreases sharply as $\mathrm{H}_{2}$ increases. When $\mathrm{H}_{2}$ increases, $\mathrm{C}_{\mathrm{gs}}$ shows an upward trend. In Figure $5 \mathrm{c}$. As $\mathrm{H}_{2}$ increases, the saturation current shows a linear decline. When $\mathrm{H}_{2}$ is less than $0.05 \mu \mathrm{m}$, the PAE increases as $\mathrm{H}_{2}$ increases, and when $\mathrm{H}_{2}$ is greater than $0.05 \mu \mathrm{m}$, the PAE decreases as $\mathrm{H}_{2}$ increases. The PAE reaches the maximum at $H_{2}=0.05 \mu \mathrm{m}$. 


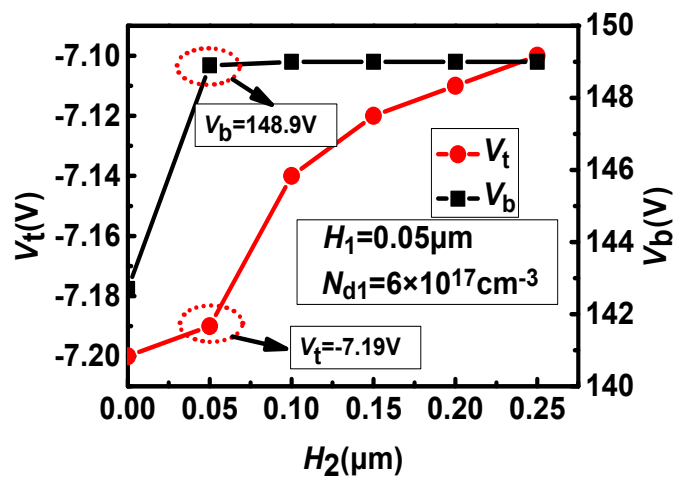

(a)

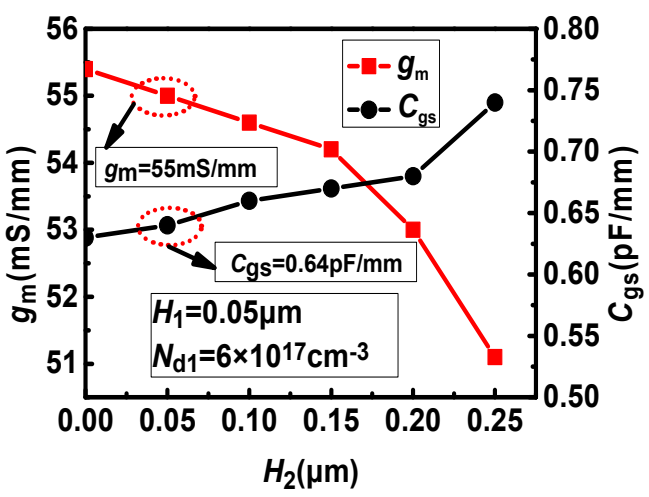

(b)

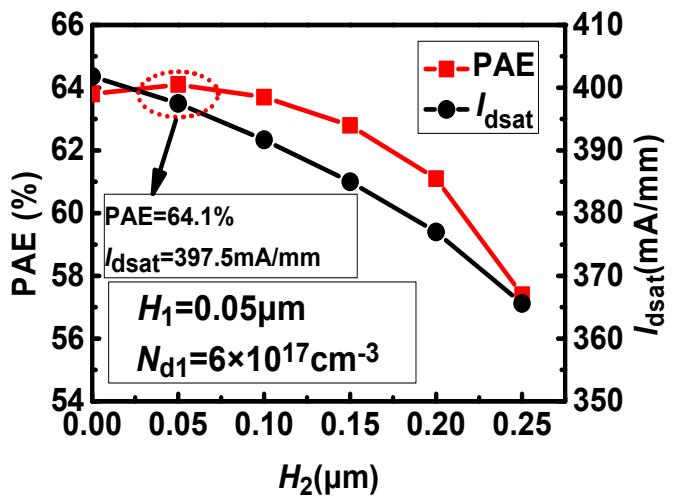

(c)

Figure 5. The effect of $\mathrm{H}_{2}$ on the device parameters: (a) $V_{\mathrm{t}}, V_{\mathrm{b}}-\mathrm{H}_{2},(\mathbf{b}) g_{\mathrm{m}}, \mathrm{C}_{\mathrm{gs}}-\mathrm{H}_{2}$, (c) PAE, $\mathrm{I}_{\mathrm{dsat}}-\mathrm{H}_{2}$.

\subsection{Mechanism to Improve Device Parameters}

Through the analysis of Figures 4 and 5, it can be acquired that when $H_{1}=0.05 \mu \mathrm{m}, H_{2}=0.05 \mu \mathrm{m}$, $N_{\mathrm{d}}=6 \times 10^{17} \mathrm{~cm}^{-3}$, the power added efficiency reaches the maximum value of $64.1 \%$. It can be seen from Equation (1) that PAE is an efficiency that considers DC and AC parameters [18]. The parameters $V_{\mathrm{t}}, g_{\mathrm{m}}$ and $C_{\mathrm{gs}}$ simulated above have an impact on power added efficiency. The larger the absolute value of the threshold voltage of the device, the more difficult it is that the gate voltage is pinched off, which results in higher DC power consumption and smaller PAE. As the maximum transconductance of the device increases, the power-added efficiency of the device tends to decrease linearly. This is because when the device transconductance increases, it means that the ability of the gate to control the current is increased, and the channel resistance is increased, resulting in an increase in the DC power consumption of the device, and the power added efficiency of the device is reduced. The larger the $C_{\mathrm{gs}}$, the greater the energy lost when charging and discharging the capacitor, resulting in a larger $P_{\mathrm{dc}}$, which in turn reduces the PAE. The LDUS-MESFET has a smaller threshold voltage and capacitance than the DRUS-MESFET. Although the transconductance is improved compared with the DRUS-MESFET, it can be seen from the simulation that the threshold voltage $V_{\mathrm{t}}$ and $C_{\mathrm{gs}}$ have a greater influence on the PAE than the transconductance. So the PAE of the LDUS-MESFET is $85.8 \%$ higher than the DRUS-MESFET.

$$
\mathrm{PAE}=\frac{P_{\mathrm{out}}-P_{\text {in }}}{P_{\mathrm{dc}}}
$$

where $P_{\text {out }}$ is output power, $P_{\text {in }}$ is input power and $P_{\mathrm{dc}}$ is DC power.

It can be seen from Table 2 that the saturation current is increased by $27.4 \%$ compared with the DRUS-MESFET. It can be obtained from Equation (2) that the saturation current is proportional to the amount of charge in the channel and the effective thickness of the channel [14]. Heavy doping 
under the lower gate and reducing the thickness of the undoped region in the channel increase the saturation current. The breakdown occurs at the edge of the gate near the drain side, and the thickness of the undoped region has a certain influence on the breakdown voltage. So the LDUS-MESFET has a lower breakdown voltage than the DRUS-MESFET. But the range of reduction is acceptable. The transconductance reflects the gate voltage's ability to control the channel current of the device. The partly high doping under the lower gate makes the transconductance of the LDUS-MESFET larger than the DRUS-MESFET. The threshold voltage refers to the gate-source voltage when the channel is pinched off. Since the LDUS-MESFET has a longitudinal concentration gradient in the channel, the concentration gradient produces a longitudinal electric field that weakens the pinch-off voltage, which results in a decrease in the absolute value of the threshold voltage. So the LDUS-MESFET has a smaller threshold voltage than the DRUS-MESFET. Compared with the DRUS-MESFET, the LDUS-MESFET has a reduced thickness of the undoped region near the drain side of the gate. So that the drain side depletion layer can be expanded and the source side expansion is reduced, this makes $C_{\mathrm{gs}}$ decrease.

$$
I_{\mathrm{dsat}}=Q(x) v(x)=\mathrm{Zb}(x) \mathrm{q} n(x) v(x)
$$

where $Z$ is the channel width, $b(x)$ is the effective depth of the channel, $\mathrm{q}$ is the electron charge, $n(x)$ is the electron density, and $v(x)$ is the electron velocity.

Table 2. Comparison of performance parameters of the two structures.

\begin{tabular}{ccc}
\hline Parameters & DRUS-MESFET & LDUS-MESFET \\
\hline$I_{\mathrm{dsat}}(\mathrm{mA} / \mathrm{mm})$ & 312 & 397.5 \\
$V_{\mathrm{b}}(\mathrm{V})$ & 156.9 & 148.9 \\
$g_{\mathrm{m}}(\mathrm{mS} / \mathrm{mm})$ & 43.8 & 55 \\
$V_{\mathrm{t}}(\mathrm{V})$ & -10.1 & -7.19 \\
$C_{\mathrm{gs}}(\mathrm{pF} / \mathrm{mm})$ & 0.74 & 0.64 \\
PAE $(\%)$ & 34.5 & 64.1 \\
\hline
\end{tabular}

\section{Conclusions}

An improved DRUS 4H-SiC MESFET with layered doping under lower gate is proposed and simulated in this paper to increase the PAE of the device. On the basis of the DRUS-MESFET, in order to maximize the power added efficiency, an upper layer region of $0.05 \mu \mathrm{m}$ thick is introduced under the lower gate, and the thickness of the partly undoped region of the DRUS-MESFET was optimized. The structure achieves a PAE of $64.1 \%$, which is $85.8 \%$ larger than the DRUS-MESFET. In addition, the LDUS-MESFET has a certain improve in saturation current, threshold voltage, transconductance, and gate-source capacitance. Although the breakdown voltage is reduced, the drop value is within the acceptable range. Overall, the LDUS-MESFET has wider application in the radio frequency direction.

Author Contributions: Data curation, X.W.; formal analysis, T.Z.; project administration, H.J.; writing-original draft, Y.L.; writing-review and editing, T.L., Y.T., S.Z., and Y.Y. All authors have read and agreed to the published version of the manuscript.

Funding: This work was supported by the National Natural Science Foundation of China (NSFC) under Grant No. 61671343.

Conflicts of Interest: The authors declare no conflict of interest.

\section{References}

1. Hjelmgren, H.; Allerstam, F.; Andersson, K. Transient simulation of microwave SiC MESFET with improved trap models. IEEE Trans. Electron. Devices 2012, 57, 729-732. [CrossRef]

2. Zhang, Y.R.; Zhang, B.; Li, Z.J. Two-dimensional analysis of the interface state effect on current gain for a 4H-SiC bipolar junction transistor. Chin. Phys. B 2010, 19. [CrossRef] 
3. Chen, F.-P.; Zhang, Y.-M.; Zhang, Y.-M.; Lu, H.-L.; Song, Q.-W. Simulation research on offset field-plate used as edge termination in $4 \mathrm{H}-\mathrm{SiC}$ merged PiN-Schottky diodes. Chin. Phys. B 2010, 19. [CrossRef]

4. Zhang, X.J.; Yang, Y.T.; Duan, B.X.; Chen, B.; Chai, C.-C.; Song, K. New 4H silicon carbide metal semiconductor field-effect transistor with a buffer layer between the gate and the channel layer. Chin. Phys. B 2012, 21. [CrossRef]

5. Zhu, C.L.; Zhao, P.; Xia, J.H. Characterization of SiC MESFETs with narrow channel layer. Microelectron. Eng. 2006, 83, 72-74.

6. Raynaud, C.; Tournier, D.; Morel, H.; Planson, D. Comparison of high voltage and high temperature performances of wide bandgap semiconductors for vertical power devices. Diam. Relat. Mater. 2010, 19, 1-6. [CrossRef]

7. Dimitrijev, S.; Han, J.; Moghadam, H.A.; Aminbeidokhti, A. Power-switching applications beyond silicon: Status and future prospects of $\mathrm{SiC}$ and GaN devices. MRS Bull. 2015, 40, 399-405. [CrossRef]

8. Jia, H.J.; Wu, Q.Y.; Hu, M. A novel $4 \mathrm{H}-\mathrm{SiC}$ MESFET with multi-recessed p-buffer layer for high energy-efficiency applications. Superlattices Microstruct. 2017, 112, 97-104. [CrossRef]

9. Jia, H.; Wu, Q.; Hu, M.; Yang, Z.; Ma, P.; Luo, Y.; Yang, Y. An Improved UU-MESFET with High Power Added Efficiency. Micromachines 2018, 9, 573. [CrossRef] [PubMed]

10. Zhu, S.; Jia, H.; Wang, X.; Liang, Y.; Tong, Y.; Li, T.; Yang, Y. Improved MRD 4H-SiC MESFET with High Power Added Efficiency. Micromachines 2019, 10, 479. [CrossRef] [PubMed]

11. Jia, H.; Tong, Y.; Li, T.; Zhu, S.; Liang, Y.; Wang, X.; Zeng, T.; Ynag, Y. An Improved 4H-SiC MESFET with a Partially Low Doped Channel. Micromachines 2019, 10, 555. [CrossRef] [PubMed]

12. Orouji, A.A.; Aminbeidokhti, A. A novel double-recessed 4H-SiC MESFET with partly undoped space region. Superlattices Microstruct. 2011, 50, 680-690. [CrossRef]

13. Zhu, C.L.; Rusli; Tin, C.C.; Zhang, G.H.; Yoon, S.F.; Ahn, J. Improved performance of SiC MESFETs using double-recessed structure. Microelectron. Eng. 2006, 83, 92-95. [CrossRef]

14. Sze, S.M.; Ng, K.K. Physics of Semiconductor Devices, 3rd ed.; John Wiley \& Sons: Hoboken, NJ, USA, 2007; pp. 303-304, 386-398.

15. Weitzel, C.E.; Palmour, J.W.; Carter, C.H. $4 \mathrm{H}-\mathrm{SiC}$ MESFET with $2.8 \mathrm{~W} / \mathrm{mm}$ power density at $1.8 \mathrm{GHz}$. IEEE Electron. Device Lett. 1994, 15, 406-408. [CrossRef]

16. Andersson, K.; Sudow, M.; Nilsson, P.A. Fabrication and characterization of field-plated buried-gate SiC MESFETs. IEEE Electron. Device Lett. 2006, 27, 573-575. [CrossRef]

17. Razavi, S.M.; Zahiri, S.H.; Hosseini, S.E. A novel 4H-SiC MESFET with recessed gate and channel. Superlattices Microstruct. 2013, 60, 516-523. [CrossRef]

18. Lee, T.H. The Design of CMOS Radio-Frequency Integrated Circuits; Cambridge University Press: Cambridge, UK, 1998.

(C) 2019 by the authors. Licensee MDPI, Basel, Switzerland. This article is an open access article distributed under the terms and conditions of the Creative Commons Attribution (CC BY) license (http://creativecommons.org/licenses/by/4.0/). 